\title{
ON ADDITIVE AND MULTIPLICATIVE (CONTROLLED) POISSON EQUATIONS
}

\author{
G. B. DI MASI \\ Dipartimento di Matematica Pura ed Applicata, Università di Padova \\ Via Belzoni 7, 35131 Padova, Italy \\ and CNR-LADSEB \\ E-mail: dimasi@math.unipd.it \\ Ł. STETTNER \\ Institute of Mathematics \\ Polish Academy of Sciences \\ Śniadeckich 8, 00-956 Warszawa, Poland \\ E-mail: stettner@impan.gov.pl
}

\begin{abstract}
Assuming that a Markov process satisfies the minorization property, existence and properties of the solutions to the additive and multiplicative Poisson equations are studied using splitting techniques. The problem is then extended to the study of risk sensitive and risk neutral control problems and corresponding Bellman equations.
\end{abstract}

1. Introduction. On a probability space $(\Omega, \mathcal{F}, P)$ consider a Markov process $X=\left(x_{n}\right)$ taking values on a complete separable metric state space $E$ endowed with the Borel $\sigma$-algebra $\mathcal{E}$. Assume that $\left(x_{n}\right)$ has a transition operator $P\left(x_{n}, \cdot\right)$ at time $n$, i.e., for $D \in \mathcal{E}$ we have $P\left\{x_{n+1} \in D \mid x_{0}, x_{1}, \ldots, x_{n}\right\}=P\left(x_{n}, D\right), P$ a.e. Let $c: E \rightarrow \mathbb{R}$ be continuous and bounded and $\gamma>0$. We would like to find constants $\lambda$ and $\lambda_{\gamma}$ such that the functions

$$
w(x):=E_{x}\left\{\sum_{i=0}^{\infty}\left(c\left(x_{i}\right)-\lambda\right)\right\}
$$

2000 Mathematics Subject Classification: Primary 93E20; Secondary 60J05, 93C55.

Key words and phrases: risk neutral and risk sensitive control, discrete time Markov processes, splitting, Poisson equations, Bellman equations.

Research of Ł. Stettner supported by KBN grant no. 1 P03A 01328.

The paper is in final form and no version of it will be published elsewhere. 
and

$$
e^{w_{\gamma}(x)}:=E_{x}\left\{\exp \left\{\sum_{i=0}^{\infty} \gamma\left(c\left(x_{i}\right)-\lambda_{\gamma}\right)\right\}\right\}
$$

are well defined.

The problems above are closely related to the existence of solutions: a constant $\lambda$ and a function $w$ or constant $\lambda_{\gamma}$ and function $w_{\gamma}$ respectively to the following equations: additive Poisson equation (APE)

$$
w(x)+\lambda=c(x)+P w(x)
$$

where $P f(x):=E_{x}\{f(x(1))\}=\int_{E} f(y) P(x, d y)$, or multiplicative Poisson equation (MPE)

$$
e^{w_{\gamma}(x)+\lambda_{\gamma}}=e^{\gamma c(x)} \int_{E} e^{w_{\gamma}(y)} P(x, d y)
$$

respectively. In fact the functions $w$ and $w_{\gamma}$ defined in (1) and (2) are natural candidates for solutions to (3) and (4) respectively.

A sufficient condition for existence of solutions to APE is (see [9] and [5]) uniform ergodicity of $\left(x_{n}\right)$, i.e.

$$
\sup _{A \in \mathcal{E}} \sup _{x, z \in E}|P(x, A)-P(z, A)|<1 .
$$

In the case of MPE a sufficient condition for the existence of solutions can be formulated as follows (see [2] and [5])

$$
\sup _{x, z \in E} h(P(x, \cdot), P(z, \cdot))<\infty
$$

where $h(\mu, \nu):=\sup _{A, B \in \mathcal{E}} \ln \frac{\mu(A) \nu(B)}{\nu(A) \mu(B)}$ is the so called Hilbert norm in the space $\mathcal{P}(E)$ of probability measures on $E$.

In the paper we shall formulate more general conditions than (5) and (6) for the existence of solutions of APE and MPE and study limit behavior of the solutions to MPE with risk factor $\gamma$ converging to 0 .

We will be furthermore interested in the control of a Markov process. For this purpose we shall assume that $\left(x_{n}\right)$ has a controlled transition operator $P^{a_{n}}\left(x_{n}, \cdot\right)$ at time $n$, where $a_{n}$ is the control at time $n$ taking values on a compact metric space $U$ and adapted to the $\sigma$-algebra $\sigma\left\{x_{0}, x_{1}, \ldots, x_{n}\right\}$.

Let now $c: E \times U \rightarrow \mathbb{R}$ be continuous bounded. We are looking for a control $\left(a_{n}\right)$ minimizing the following cost functionals: risk neutral (average cost per unit time)

$$
J\left(\left(a_{n}\right)\right):=\limsup _{n \rightarrow \infty} \frac{1}{n} E_{x}^{\left(a_{n}\right)}\left\{\sum_{i=0}^{n-1} c\left(x_{i}, a_{i}\right)\right\}
$$

or risk sensitive cost functional

$$
J_{\gamma}\left(\left(a_{n}\right)\right):=\frac{1}{\gamma} \limsup _{n \rightarrow \infty} \frac{1}{n} \ln E_{x}^{\left(a_{n}\right)}\left\{\exp \left\{\sum_{i=0}^{n-1} \gamma c\left(x_{i}, a_{i}\right)\right\}\right\},
$$

where $E_{x}^{\left(a_{n}\right)}$ stands for expectation with respect to the conditional probability measure under control sequence $\left(a_{n}\right)$, given the initial state $x$ of the controlled process $\left(x_{n}\right)$. 
The study of risk sensitive functional is motivated by the fact that it measures not only the average cost but also higher moments of the average cost in particular its variance with weight $\gamma$ (see [1] for financial motivation of these kind of problems). It can be also considered as a dual problem to the minimization of the probability that the average cost is greater that a given benchmark (see [7]).

The following Bellman equations correspond to the cost functionals (7) and (8) respectively

$$
w(x)+\lambda=\inf _{a \in U}\left(c(x, a)+P^{a} w(x)\right)
$$

where $P^{a} f(x):=\int_{E} f(y) P^{a}(x, d y)$, and

$$
e^{w_{\gamma}(x)+\gamma \lambda_{\gamma}}=\inf _{a \in U}\left(e^{\gamma c(x, a)} \int_{E} e^{w_{\gamma}(y)} P^{a}(x, d y)\right) .
$$

One can expect that $\lambda$ and $\lambda_{\gamma}$ are optimal values of the cost functionals (7) and (8) respectively.

In what follows we shall assume the following Feller property

$(\mathrm{F}): U \times E \ni(a, x) \mapsto P^{a} f(x)$ is continuous for $f \in C(E)$.

Under (F) and controlled uniform ergodicity of the form

$$
\sup _{A \in \mathcal{E}} \sup _{a, a^{\prime} \in U} \sup _{x, z \in E}\left|P^{a}(x, A)-P^{a^{\prime}}(z, A)\right|<1
$$

there is (see [9]) a bounded continuous function $w$ and a unique constant $\lambda$ which solve the Bellman equation (9). Furthermore

$$
\lambda=\inf _{\left(a_{n}\right)} J\left(\left(a_{n}\right)\right)=J\left(\hat{u}\left(x_{n}\right)\right),
$$

where $\hat{u}$ is a Borel measurable function for which the infimum on the right hand side of (9) is attained.

If additionally to $(\mathrm{F})$ we have that

$$
\sup _{x, z \in E} \sup _{a, a^{\prime} \in U} h\left(P^{a}(x, \cdot), P^{a^{\prime}}(z, \cdot)\right)<\infty
$$

then there exist (see [2]) a bounded function $w_{\gamma}$ and a unique constant $\lambda_{\gamma}$ for which the Bellman equation (10) is satisfied. Moreover

$$
\lambda_{\gamma}=\inf _{\left(a_{n}\right)} J_{\gamma}\left(\left(a_{n}\right)\right)=J_{\gamma}\left(\left(\hat{u}_{\gamma}\left(x_{n}\right)\right)\right),
$$

where $\hat{u}_{\gamma}$ is a function for which the infimum in the right hand side of (10) is attained.

We shall consider the following two classes of controls: Markov controls $\mathcal{U}_{M}=\left\{\left(a_{n}\right)\right.$ : $\left.a_{n}=u_{n}\left(x_{n}\right)\right\}$, where $u_{n}: E \rightarrow U$, and stationary controls $\mathcal{U}_{s}=\left\{\left(a_{n}\right): a_{n}=u(x(n))\right\}$, where $u: E \rightarrow U$. We shall also indentify a Markov control $a_{n}=u_{n}\left(x_{n}\right)$ with the sequence $\left(u_{n}\right)$ of Borel measurable functions $u_{n}: E \rightarrow U$. Similarly a stationary control $a_{n}=u\left(x_{n}\right)$ shall be identified with the Borel measurable function $u: E \rightarrow U$.

In the paper we generalize results concerning the existence of solutions to additive and multiplicative uncontrolled and controlled Poisson equations. The assumptions (5), (6) and (11), (13) are mainly satisfied when the state space is compact. Our purpose is to show the existence results under assumptions satisfied by ergodic processes on locally 
compact spaces. Since we shall use the so called splitting technique of Markov processes introduced in [6] we shall assume the following minorization property:

(A1) $\exists_{\beta>0} \quad \exists_{C \text { compact } \in \mathcal{E}} \quad \exists_{\nu \in \mathcal{P}(E)}$ with $\nu(C)=1$ such that $\forall_{A \in \mathcal{E}}$

$$
\inf _{x \in C} \inf _{a \in U} P^{a}(x, A) \geq \beta \nu(A) .
$$

Furthermore the following ergodicity assumption will be considered

(A2) $C$ in (A1) is ergodic, i.e. $\forall_{\left(a_{n}\right) \in \mathcal{U}_{M}} \forall_{x \in E} E_{x}^{\left(a_{n}\right)}\left\{\tau_{C}\right\}<\infty$, where $\tau_{C}=\inf \{i>0$ : $\left.x_{i} \in C\right\}$ and $\forall\left(a_{n}\right) \in \mathcal{U}_{M}$

$$
\sup _{x \in C} E_{x}^{\left(a_{n}\right)}\left\{\tau_{C}\right\}<\infty .
$$

Notice that assumptions (A1) and (A2) are roughly satisfied by nondegenerate ergodic processes on locally compact state space $E$, where $C$ is a ball which is a positive recurrent set. Given the set $C$ satisfying (A1) and (A2) and the Markov control $\left(u_{n}\right)$ we consider a new state space $\hat{E}=\{C \times\{0\} \cup C \times\{1\} \cup(E \backslash C) \times\{0\}\}$ and splitting of $\left(x_{n}\right)$ in the form $\hat{x}_{n}=\left(x_{n}^{1}, x_{n}^{2}\right) \in \hat{E}$ with Markov control of the form $a_{n}=u_{n}\left(x_{n}^{1}\right)$ and dynamics defined in the following way:

(i) when $\left(x_{n}^{1}, x_{n}^{2}\right) \in C \times\{0\}, x_{n}^{1}$ moves to $y$ accordingly to $(1-\beta)^{-1}\left(P^{a_{n}}\left(x_{n}^{1}, d y\right)-\right.$ $\beta \nu(d y))$ and whenever $y \in C, x_{n}^{2}$ is changed into $x_{n+1}^{2}=\beta_{n+1}$, where $\beta_{n}$ is i.i.d. $P\left\{\beta_{n}=0\right\}=1-\beta, P\left\{\beta_{n}=1\right\}=\beta$,

(ii) when $\left(x_{n}^{1}, x_{n}^{2}\right) \in C \times\{1\}, x_{n}^{1}$ moves to $y$ accordingly to $\nu$ and $x_{n+1}^{2}=\beta_{n+1}$,

(iii) when $\left(x_{n}^{1}, x_{n}^{2}\right) \in(E \backslash C) \times\{0\}, x_{n}^{1}$ moves to $y$ accordingly to $P^{a_{n}}\left(x_{n}^{1}, d y\right)$ and whenever $y \in C, x_{n}^{2}$ is changed into $x_{n+1}^{2}=\beta_{n+1}$.

Let $C_{0}=C \times\{0\}, C_{1}=C \times\{1\}$. The following properties of the split Markov process are shown in [3]

Lemma 1. Under Markov control $\left(a_{n}\right) \in \mathcal{U}_{M}$ the process $\left(\hat{x}_{n}=\left(x_{n}^{1}, x_{n}^{2}\right)\right)$ is Markov with transition operator $\hat{P}^{a_{n}}\left(\hat{x}_{n}, d y\right)$ defined by (i)-(iii) and has a unique invariant measure $\Psi^{\left(a_{n}\right)}$ given by the formula

$$
\Psi^{\left(a_{n}\right)}(A)=\frac{\hat{E}_{z}^{\left(a_{n}\right)}\left\{\sum_{i=1}^{\tau_{C_{1}}} \chi_{A}\left(\hat{x}_{i}\right)\right\}}{\hat{E}_{z}^{\left(a_{n}\right)}\left\{\tau_{C_{1}}\right\}},
$$

with $z \in C_{1}$, for any Borel subset $A$ of $\hat{E}$, where $E_{z}^{\left(a_{n}\right)}$ stands for the conditional law of the Markov process $\hat{x}_{n}$ with initial state $z$. Furthermore the first coordinate $\left(x_{n}^{1}\right)$ is also a Markov process with transition operator $P^{a_{n}}\left(x_{n}^{1}, d y\right)$.

Corollary 1. For any bounded Borel measurable function $f: E^{m} \rightarrow \mathbb{R}, m=1,2, \ldots$, and control $\left(a_{n}\right) \in \mathcal{U}_{M}$ we have

$$
E_{x}^{\left(a_{n}\right)}\left\{f\left(x_{1}, x_{2}, \ldots, x_{m}\right)\right\}=\hat{E}_{\delta_{x}^{*}}^{\left(a_{n}\right)}\left\{f\left(x_{1}^{1}, x_{2}^{1}, \ldots, x_{m}^{1}\right)\right\}
$$

where $\delta_{x}^{*}=\delta_{(x, 0)}$ for $x \in E \backslash C$ and $\delta_{x}^{*}=(1-\beta) \delta_{(x, 0)}+\beta \delta_{(x, 1)}$ for $x \in C$.

The paper consists of 8 sections. We formulate first the existence results for APE which generalize results of [4] proved there in a more specific case and recall results on MPE from [3]. In section 4 we show that APE can be considered as the limit of MPEs letting the risk factor $\gamma$ tend to 0 . Starting from section 5 we consider a uniformly ergodic 
approximation of Markov processes. An approximating process with transition operator $P_{N}^{a}(x, d y)$ satisfies assumptions (11) and (13), so that we have the existence of solutions to controlled APE and MPE. In section 6 using the above approximation we show the existence of solutions to APE for processes satisfying (A1) and (A2). In section 7 we recall the existence results for controlled MPE from [3] and finally in section 8 we consider the asymptotics of MPE for the risk factor $\gamma$ converging to 0.

2. The study of additive Poisson equation. We start with an obvious lemma which follows directly from the boundedness of $c$, and conditions (A1) and (A2)

Lemma 2. Given Borel measurable $u: E \rightarrow U$ there is a unique $\lambda(u)$ such that for $x \in C_{1}$

$$
\hat{E}_{x}\left\{\sum_{i=1}^{\tau_{C_{1}}}\left(c\left(x_{i}^{1}, u\left(x_{i}^{1}\right)\right)-\lambda(u)\right)\right\}=0 .
$$

Furthermore $\lambda(u)=\int_{\hat{E}} c\left(x^{1}, u\left(x^{1}\right)\right) \Psi^{u}(d x)$.

For Borel measurable $u: E \rightarrow U$ let

$$
\hat{w}^{u}(x):=\hat{E}_{x}\left\{\sum_{i=0}^{\tau_{C_{1}}}\left(c\left(x_{i}^{1}, u\left(x_{i}^{1}\right)\right)-\lambda(u)\right)\right\} .
$$

In analogy to [4] (see also [3]), where more specific case was studied, we can show the following results:

LEMma 3. The function $\hat{w}^{u}$ is a solution to the additive Poisson equation (APE) for the split Markov process $\left(\hat{x}_{n}\right)$ :

$$
\hat{w}^{u}(x)=c\left(x^{1}, u\left(x^{1}\right)\right)-\lambda(u)+\int_{\hat{E}} \hat{w}^{u}(y) \hat{P}^{u\left(x^{1}\right)}(x, d y) .
$$

Furthermore, if $\hat{w}$ and $\lambda$ satisfy the equation

$$
\hat{w}(x)=c\left(x^{1}, u\left(x^{1}\right)\right)-\lambda+\int_{\hat{E}} \hat{w}(y) \hat{P}^{u\left(x^{1}\right)}(x, d y)
$$

and $\hat{w}$ is integrable with respect to the measure $\Psi^{u}$ then $\lambda=\lambda(u)$ (defined in Lemma 2) and $\hat{w}$ differs from $\hat{w}^{u}$ by an additive constant $\Psi^{u}$ almost everywhere.

Corollary 2. Given a solution $\tilde{w}^{u}: \hat{E} \rightarrow \mathbb{R}$ to APE we have that $w^{u}$ defined by

$$
w^{u}(x):=\tilde{w}^{u}(x, 0)+1_{C}(x) \beta\left(\tilde{w}^{u}(x, 1)-\tilde{w}^{u}(x, 0)\right)
$$

is a solution to APE for the original Markov process $\left(x_{n}\right)$

$$
w^{u}(x)=c(x, u(x))-\lambda(u)+\int_{E} w^{u}(y) P^{u(x)}(x, d y) .
$$

Furthermore if $w^{u}$ is a solution to (22) then $\tilde{w}^{u}$ defined by

$$
\tilde{w}^{u}\left(x^{1}, x^{2}\right)=c\left(x^{1}, u\left(x^{1}\right)\right)-\lambda(u)+\hat{E}_{x^{1}, x^{2}}\left\{w^{u}\left(x^{1}(1)\right)\right\}
$$

is a solution to (19). 
Proposition 1. For Borel measurable $u: E \rightarrow U$ the value $\lambda(u)$ defined in Lemma 2 is equal to

$$
\lambda(u)=\lim _{n \rightarrow \infty} \frac{1}{n} E_{x}\left\{\sum_{i=0}^{n-1} c\left(x_{i}, u\left(x_{i}\right)\right)\right\}
$$

3. The study of the multiplicative Poisson equation. To study the MPE we need a stronger assumption than (A2). Fix $\gamma>0$. We shall impose the condition that

(A3) $\forall_{\left(a_{n}\right) \in \mathcal{U}_{s}} \exists_{d}$ s.t. $\forall_{x \in \hat{E}}$

$$
\hat{E}_{x}^{\left(a_{n}\right)}\left\{\exp \left\{\sum_{i=1}^{\tau_{C_{1}}} \gamma\left(c\left(x_{i}^{1}, a_{i}\right)-d\right)\right\}\right\}<\infty
$$

and for $x \in C_{1}$

$$
\hat{E}_{x}^{\left(a_{n}\right)}\left\{\exp \left\{\sum_{i=1}^{\tau_{C_{1}}} \gamma\left(c\left(x_{i}^{1}, a_{i}\right)-d\right)\right\}\right\} \geq 1 .
$$

Under (A3) we easily obtain

LEMma 4. Under (A3) for Borel measurable $u: E \rightarrow U$ there is a unique $\lambda_{\gamma}(u)$ such that

$$
\hat{E}_{x}^{\left(a_{n}\right)}\left\{\exp \left\{\sum_{i=1}^{\tau_{C_{1}}} \gamma\left(c\left(x_{i}^{1}, a_{i}\right)-\lambda_{\gamma}(u)\right)\right\}\right\}=1
$$

for $x \in C_{1}$.

For Borel measurable $u: E \rightarrow U$ and $\gamma>0$ for which (A3) holds define

$$
e^{\hat{w}_{\gamma}^{u}(x)}=\hat{E}_{x}^{u}\left\{\exp \left\{\sum_{i=0}^{\tau_{C_{1}}} \gamma\left(c\left(x_{i}^{1}, u\left(x_{i}^{1}\right)\right)-\lambda_{\gamma}(u)\right)\right\}\right\},
$$

For a Borel measurable function $\tilde{w}: \hat{E} \rightarrow \mathbb{R}$ define the operator $\Phi(\tilde{w})$ by the formula

$$
e^{\Phi(\tilde{w})}=(1-\beta) \int_{C} e^{\tilde{w}(x, 0)} \nu(d x)+\beta \int_{C} e^{\tilde{w}(x, 1)} \nu(d x)
$$

whenever it is well defined. We have (see [3] for the proofs)

Lemma 5. The function $\hat{w}_{\gamma}^{u}$ defined in (26) is a solution to the multiplicative Poisson equation (MPE) for the split Markov process $\left(\hat{x}_{n}\right)$ :

$$
e^{\hat{w}_{\gamma}^{u}(x)}=e^{\gamma c\left(x^{1}, u\left(x^{1}\right)\right)-\lambda_{\gamma}(u)} \int_{\hat{E}} e^{\hat{w}_{\gamma}^{u}(y)} \hat{P}^{u\left(x^{1}\right)}(x, d y) .
$$

Moreover $\Phi\left(\hat{w}_{\gamma}^{u}\right)=0$, and for any other solution $\tilde{w}^{u}$ to (28) we have

$$
\tilde{w}^{u}(x)-\Phi\left(\tilde{w}^{u}\right) \geq \hat{w}_{\gamma}^{u}(x)
$$

with equality for $\Psi^{u}$ almost all $x \in \hat{E}$. Furthermore, if $\tilde{w}$ and $\lambda$ satisfy the equation

$$
e^{\tilde{w}(x)}=e^{\gamma c\left(x^{1}, u\left(x^{1}\right)\right)-\lambda} \int_{\hat{E}} e^{\tilde{w}(y)} \hat{P}^{u\left(x^{1}\right)}(x, d y)
$$

then $\lambda \geq \lambda_{\gamma}(u)$. 
Corollary 3. For $x \in E$ and a solution $\tilde{w}^{u}: \hat{E} \rightarrow \mathbb{R}$ and $\lambda$ to MPE (28) we have that $w^{u}$ defined by

$$
e^{w^{u}(x)}:=e^{\tilde{w}^{u}(x, 0)}+1_{C}(x) \beta\left(e^{\tilde{w}^{u}(x, 1)}-e^{\tilde{w}^{u}(x, 0)}\right)
$$

is a solution to MPE for the original Markov process $\left(x_{n}\right)$

$$
e^{w^{u}(x)}=e^{\gamma c(x, u(x))-\lambda} \int_{E} e^{w^{u}(y)} P^{u(x)}(x, d y) .
$$

Furthermore if $w^{u}$ is a solution to (32) then $\tilde{w}^{u}$ defined by

$$
e^{\tilde{w}^{u}\left(x^{1}, x^{2}\right)}=e^{\gamma c\left(x^{1}, u\left(x^{1}\right)\right)-\lambda} \hat{E}_{x^{1}, x^{2}}\left\{e^{w^{u}\left(x_{1}^{1}\right)}\right\}
$$

is a solution to $(28)$.

From Proposition 1 of [3] we have

Proposition 2. If for Borel measurable $u: E \rightarrow U$

(D1): $\quad \hat{E}_{x}^{\left(a_{n}\right)}\left\{\exp \left\{\gamma\|c\|_{s p} \tau_{C_{1}}\right\}\right\}<\infty$ for $x \in \hat{E}$, with $a_{n}=u\left(x_{n}^{1}\right)$, where $\|c\|_{s p}:=\sup _{(x, a) \in E \times U} c(x, a)-\inf _{(x, a) \in E \times U} c(x, a)$, then for $x \in E$

$$
\left.\lambda_{\gamma}(u)=\frac{1}{\gamma} \lim _{n \rightarrow \infty} \frac{1}{n} \ln E_{x}^{u}\left\{\exp \left\{\sum_{i=0}^{n-1} \gamma c\left(x_{i}, u\left(x_{i}\right)\right)\right)\right\}\right\} .
$$

4. Asymptotics of MPEs. Given Borel measurable $u: E \rightarrow U$ assume that we have (D1) satisfied for $0<\gamma<\gamma_{0}$. Then by Proposition 2 there are solutions $\lambda_{\gamma}(u)$ and $w_{\gamma}^{u}$ to the MPE (32) and $\lambda_{\gamma}(u)$ is of the form (34). We are now interested in the limit behavior of $\lambda_{\gamma}(u)$ and $w_{\gamma}^{u}$ as $\gamma \rightarrow 0$.

Proposition 3. We have that $\lambda_{\gamma}(u)$ decreases to $\lambda(u)$ and $w_{\gamma}^{u}(x)$ converges to $w^{u}(x)$ for $U_{1}^{u}(\nu, \cdot)$ almost all $x \in E$ as $\gamma \downarrow 0$, where $\lambda(u)$ and $w^{u}$ are solutions to the APE (22) and $U_{1}^{u}(\nu, \cdot)=\sum_{i=1}^{\infty} 2^{-i} P^{u i}(\nu, \cdot)$.

Proof. Notice first that by the Hölder inequality

$$
\left.\left.\frac{1}{\gamma_{1}} \ln E_{x}^{u}\left\{\exp \left\{\sum_{i=0}^{n-1} \gamma_{1} c\left(x_{i}, u\left(x_{i}\right)\right)\right)\right\}\right\} \leq \frac{1}{\gamma_{2}} \ln E_{x}^{u}\left\{\exp \left\{\sum_{i=0}^{n-1} \gamma_{2} c\left(x_{i}, u\left(x_{i}\right)\right)\right)\right\}\right\}
$$

whenever $0<\gamma_{1} \leq \gamma_{2}$. Therefore by $(34) \lambda_{\gamma}(u)$ is decreasing as $\gamma \rightarrow 0$. Consequently $\lambda_{0}=$ $\lim _{\gamma \downarrow 0} \lambda_{\gamma}(u)$. Consider now the split Markov process $\left(\hat{x}_{n}\right)$ corresponding to a stationary control $u$. Let $\hat{w}_{\gamma}^{u}$ be given by (26). Then

$$
e^{\hat{w}_{\gamma}^{u}(x)} \leq \hat{E}_{x}^{u}\left\{\exp \left\{\sum_{i=0}^{\tau_{C_{1}}} \gamma\left(c\left(x_{i}^{1}, u\left(x_{i}^{1}\right)\right)-\lambda_{0}\right)\right\}\right\} .
$$

Consequently by de l'Hospital's rule we have

$$
\begin{aligned}
\underset{\gamma \downarrow 0}{\limsup } \hat{w}_{\gamma}^{u}(x) & \leq \limsup _{\gamma \downarrow 0} \frac{1}{\gamma} \ln \hat{E}_{x}^{u}\left\{\exp \left\{\sum_{i=0}^{\tau_{C_{1}}} \gamma\left(c\left(x_{i}^{1}, u\left(x_{i}^{1}\right)\right)-\lambda_{0}\right)\right\}\right\} \\
& =\hat{E}_{x}\left\{\sum_{i=0}^{\tau_{C_{1}}}\left(c\left(x_{i}^{1}, u\left(x_{i}^{1}\right)\right)-\lambda_{0}\right)\right\} .
\end{aligned}
$$


Similarly for $\bar{\gamma}<\gamma_{0}$

$$
\begin{aligned}
\liminf _{\gamma \downarrow 0} \frac{1}{\gamma} \hat{w}_{\gamma}(x) & \geq \liminf _{\gamma \downarrow 0} \ln \hat{E}_{x}^{u}\left\{\exp \left\{\sum_{i=0}^{\tau_{C_{1}}} \gamma\left(c\left(x_{i}^{1}, u\left(x_{i}^{1}\right)\right)-\lambda_{\bar{\gamma}}\right)\right\}\right\} \\
& =\hat{E}_{x}\left\{\sum_{i=0}^{\tau_{C_{1}}}\left(c\left(x_{i}^{1}, u\left(x_{i}^{1}\right)\right)-\lambda_{\bar{\gamma}}\right)\right\} .
\end{aligned}
$$

Therefore

$$
\begin{aligned}
\hat{E}_{x}\left\{\sum_{i=0}^{\tau_{C_{1}}}\left(c\left(x_{i}^{1}, u\left(x_{i}^{1}\right)\right)-\lambda_{\bar{\gamma}}\right)\right\} & \leq \liminf _{\gamma \downarrow 0} \frac{1}{\gamma} \hat{w}_{\gamma}(x) \\
& \leq \limsup _{\gamma \downarrow 0} \frac{1}{\gamma} \hat{w}_{\gamma}(x) \leq \hat{E}_{x}\left\{\sum_{i=0}^{\tau_{C_{1}}}\left(c\left(x_{i}^{1}, u\left(x_{i}^{1}\right)\right)-\lambda_{0}\right)\right\}
\end{aligned}
$$

and letting $\bar{\gamma} \rightarrow 0$ we obtain that

$$
\lim _{\gamma \downarrow 0} \frac{1}{\gamma} \hat{w}_{\gamma}^{u}(x)=\hat{E}_{x}\left\{\sum_{i=0}^{\tau_{C_{1}}}\left(c\left(x_{i}^{1}, u\left(x_{i}^{1}\right)\right)-\lambda_{0}\right)\right\}:=w(x) .
$$

Since $\hat{w}_{\gamma}^{u}\left(x_{0}\right)=\gamma\left(c\left(x_{0}, u\left(x_{0}\right)\right)-\lambda_{\gamma}(u)\right)$ for $x_{0} \in C_{1}$, we have $w\left(x_{0}\right)=c\left(x_{0}, u\left(x_{0}\right)\right)-\lambda_{0}$. Therefore by Lemma $3, \lambda_{0}=\lambda(u)$ and $w(x)=\hat{w}^{u}(x)$ for $\Psi^{u}$ almost all $x \in \hat{E}$. From (21), (31) and the form (15) of the invariant measure $\Psi^{u}$ we immediately have that $\lim _{\gamma \downarrow 0} w_{\gamma}^{u}(x)=w^{u}(x)$ for $U_{1}^{u}(\nu, \cdot)$ almost all $x \in E$, which completes the proof.

5. Approximations to the Markov process. In this section we shall introduce an approximation to a Markov transition operator in the form of a Markov transition operator satisfying condition (13). We assume first that

$$
P^{a}(x, A)=\int_{A} p(x, a, y) \nu(d y)
$$

where $p>0$ is a continuous function. Moreover letting $|x|:=\rho(x, \theta)$, where $\rho$ is a metric on $E$ and $\theta \in E$ is a fixed point define

$$
\tilde{p}_{N}(x, a, y)= \begin{cases}\frac{p(x, a, y)}{\Delta_{N}^{a}(x)} & \text { for }|y| \leq N \\ \frac{p(\theta, \bar{a}, y)}{\Delta_{N}^{a}(x)} & \text { for }|y| \geq N+1 \\ \frac{p(x, a, y)(N+1-|y|)+p(\theta, \bar{a}, y)(|y|-N)}{\Delta_{N}^{a}(x)} & \text { elsewhere }\end{cases}
$$

with $\Delta_{N}^{a}(x)=P^{a}\left(x, B_{N}\right)+P^{\bar{a}}\left(\theta, B_{N+1}^{c}\right)+\int_{B_{N+1} \backslash B_{N}}[p(x, a, y)(N+1-|y|)+p(\theta, \bar{a}, y)(|y|-$ $N)] \nu(d y)$, where $B_{N}=\{x \in E:|x| \leq N\}$ and $\bar{a}$ is a fixed element of $U$. Then let

$$
\begin{aligned}
& p_{N}(x, a, y)=\tilde{p}_{N}(x, a, y) \text { if }|x| \leq N \\
& \left.p_{N}(x, a, y)=\tilde{p}_{N}\left(\frac{x}{|x|} N, a, y\right)\right) \text { for }|x|>N,
\end{aligned}
$$

and define

$$
P_{N}^{a}(x, d y)=p_{N}(x, a, y) \nu(d y)
$$

We clearly have 
LEMMA 6.

$$
\sup _{a \in U}\left\|P_{N}^{a}(x, \cdot)-P^{a}(x, \cdot)\right\|_{v a r} \rightarrow 0
$$

as $N \rightarrow \infty$, uniformly in $x$ from compact sets. Furthermore for each $N$

$$
\sup _{a, a^{\prime} \in U} \sup _{x, x^{\prime} \in E} \sup _{y \in E} \frac{p_{N}(x, a, y)}{p_{N}\left(x^{\prime}, a^{\prime}, y\right)}<\infty
$$

which means that (13) is satisfied.

REMARK 1. For the controlled Markov process with transition operator $P_{N}^{a}(x, d y)$ defined in (41) we clearly have that conditions (F), (11) and (13) are satisfied. Consequently we have solutions $w^{(N)}, \lambda^{(N)}$ and $w_{\gamma}^{(N)}, \lambda_{\gamma}^{(N)}$ to the Bellman equations (9) and (10) respectively with operator $P^{a}$ replaced by $P_{N}^{a}$. Furthermore, there exist optimal stationary controls $\hat{u}^{(N)}$ and $\hat{u}_{\gamma}^{(N)}$, which are in fact selectors to the right hand sides of (9) and (10) respectively, for the cost functionals $J^{(N)}$ and $J_{\gamma}^{(N)}$ which correspond to the functionals $J$ and $J_{\gamma}$ with operator $P^{a}$ replaced by $P_{N}^{a}$.

6. Solution to additive Bellman equation. We shall need the following assumption:

(A5) $\exists_{\epsilon>0}$ such that $\forall_{K \text { compact, } \subset \hat{E}}$

$$
\sup _{a \in U} \sup _{x \in K} \sup _{N} \hat{E}_{x}^{a, N}\left\{\left|\sum_{i=1}^{\tau_{C_{1}}}\left(c\left(x_{i}^{1}, \hat{u}^{(N)}\left(x_{i}^{1}\right)\right)-\lambda^{(N)}\right)\right|^{1+\epsilon}\right\}=M(K)<\infty,
$$

where the control at the first time is $a_{0}=a$ and $a_{n}=\hat{u}^{(N)}\left(x_{n}^{1}\right)$ for $n \geq 1$.

Notice that (A5) in particular is satisfied when $\sup _{\left(a_{n}\right)} \sup _{x \in K} \hat{E}_{x}^{\left(a_{n}\right), N}\left\{\tau_{C_{1}}^{1+\epsilon}\right\}<\infty$ which is satisfied when $C$ is a positive recurrent set which the process enters no matter what kind of control is used.

THEOREM 1. Under (A5) there exist $\lambda$ and a continuous function $w: E \rightarrow \mathbb{R}$ such that

$$
w(x)=\inf _{a \in U}\left[c(x, a)-\lambda+\int_{E} w(y) P^{a}(x, d y)\right] .
$$

Moreover $\lambda$ is an optimal value of the cost functional (7) within the class of controls $u$ from $\mathcal{U}_{s}$ such that $\hat{E}_{x^{1}, x^{2}}^{u\left(x^{1}\right)}\left\{w\left(x_{1}^{1}\right)\right\}$ is $\Psi^{u}$ integrable. The control $\hat{u}$ for which the infimum in (45) is attained, is an optimal control. If for an admissible control $\left(a_{n}\right)$ we have $\lim _{t \rightarrow \infty} \frac{1}{t} E_{x}^{\left(a_{n}\right)}\left\{w\left(x_{t}\right)\right\}=0$ then $\lambda \leq J_{x}\left(\left(a_{n}\right)\right)$.

Proof. The proof consists of several steps:

Step 1. Notice first that if $\hat{w}_{N}^{\hat{u}^{(N)}}$ is a solution to APE corresponding to transition operator $P^{\hat{u}^{(N)}}$ we have

$$
\hat{E}_{x}^{a, N}\left\{1_{C_{1}}\left(\hat{x}_{1}\right) \hat{w}_{N}^{\hat{u}^{(N)}}\left(\hat{x}_{1}\right)\right\}=\hat{E}_{x}^{a, N}\left\{\chi_{C_{1}}\left(\hat{x}_{1}\right)\left(c\left(x_{1}^{1}, \hat{u}^{(N)}\left(x_{1}^{1}\right)\right)-\lambda^{(N)}\left(\hat{u}^{(N)}\right)\right)\right\}
$$

and

$$
\hat{E}_{x}^{a, N}\left\{1_{C_{1}^{c}}\left(\hat{x}_{1}\right) \hat{w}_{N}^{\hat{u}^{(N)}}\left(\hat{x}_{1}\right)\right\}=\hat{E}_{x}^{a, N}\left\{\chi_{C_{1}^{c}}\left(\hat{x}_{1}\right) \sum_{i=1}^{\tau_{C_{1}}}\left(c\left(x_{i}^{1}, \hat{u}^{(N)}\left(x_{i}^{1}\right)\right)-\lambda^{(N)}\left(\hat{u}^{(N)}\right)\right)\right\}
$$


and therefore by (A5) the terms

$$
\begin{aligned}
& f_{1}^{a, N}(x):=\hat{E}_{x}^{a, N}\left\{1_{C_{1}}\left(\hat{x}_{1}\right) \hat{w}_{N}^{\hat{u}^{(N)}}\left(\hat{x}_{1}\right)\right\} \\
& f_{2}^{a, N}(x):=\hat{E}_{x}^{a, N}\left\{1_{C_{0}}\left(\hat{x}_{1}\right) \hat{w}_{N}^{\hat{u}^{(N)}}\left(\hat{x}_{1}\right)\right\}
\end{aligned}
$$

and

$$
f_{3}^{a, N}(x):=\hat{E}_{x}^{a, N}\left\{1_{(E \backslash C) \times\{0\}}\left(\hat{x}_{1}\right) \hat{w}_{N}^{\hat{u}^{(N)}}\left(\hat{x}_{1}\right)\right\}
$$

are bounded in $N$ uniformly on compact subsets of $\left(E_{0} \cup C_{1}\right) \times U$.

Step 2. We show now that for $N=1,2, \ldots$, the functions $f_{1}^{a, N}(x), f_{2}^{a, N}(x)$ and $f_{3}^{a, N}(x)$ are equicontinuous in $x$ and $a$ from compact subsets of $E_{0} \cup C_{1}$ and $U$ respectively.

Notice that by (42) for each compact set $K \subset E_{0} \cup C_{1}, \varepsilon^{\prime}>0$ there is a compact set $K_{1} \supset C_{0} \cup C_{1}$ such that

$$
\sup _{a \in U} \sup _{x \in K} \sup _{N} \hat{P}_{x}^{a N}\left\{\hat{x}_{1} \in K_{1}^{c}\right\}<\varepsilon^{\prime} .
$$

Furthermore by the Hölder inequality

$$
\begin{aligned}
& \sup _{a \in U} \sup _{x \in K} \sup _{N}\left|\hat{E}_{x}^{a, N}\left\{1_{K_{1}^{c}}\left(\hat{x}_{1}\right) \sum_{i=1}^{\tau_{C_{1}}}\left(c\left(x_{i}^{1}, \hat{u}^{(N)}\left(x_{i}^{1}\right)\right)-\lambda^{N}\left(\hat{u}^{(N)}\right)\right)\right\}\right| \\
& \leq \sup _{a \in U} \sup _{x \in K} \sup _{N}\left(\hat{P}_{x}^{a, N}\left\{\hat{x}_{1} \in K_{1}^{c}\right\}\right)^{\frac{\varepsilon}{1+\varepsilon}} \sup _{a \in U} \sup _{x \in K} \sup _{N}\left(\hat { E } _ { x } ^ { a , N } \left\{\mid \sum_{i=1}^{\tau_{C_{1}}}\left(c\left(x_{i}^{1}, \hat{u}^{N}\left(x_{i}^{1}\right)\right)\right.\right.\right. \\
& \left.\left.\left.-\lambda^{N}\left(\hat{u}_{N}\right)\right)\left.\right|^{(1+\epsilon)}\right\}\right)^{\frac{1}{1+\varepsilon}} \leq \varepsilon^{\frac{\varepsilon}{1+\varepsilon}}(M(K))^{\frac{1}{1+\varepsilon}} .
\end{aligned}
$$

Now for $a, a^{\prime} \in U, x, x^{\prime} \in \hat{E}$

$$
\begin{aligned}
& \left|f_{1}^{a, N}(x)-f_{1}^{a^{\prime}, N}\left(x^{\prime}\right)\right| \leq\|c\|\left\|\hat{P}^{a N}\left(x, C_{1} \cap \cdot\right)-\hat{P}^{a^{\prime} N}\left(x^{\prime}, C_{1} \cap \cdot\right)\right\|_{\text {var }}, \\
& \left|f_{2}^{a, N}(x)-f_{2}^{a^{\prime}, N}\left(x^{\prime}\right)\right| \leq \sup _{x \in C_{0}}\left|\hat{w}_{N}^{\hat{u}^{(N)}}(x)\right|\left\|\hat{P}^{a N}\left(x, C_{0} \cap \cdot\right)-\hat{P}^{a^{\prime} N}\left(x^{\prime}, C_{0} \cap \cdot\right)\right\|_{\text {var }}
\end{aligned}
$$

and using (47)

$$
\begin{array}{r}
\left|f_{3}^{a, N}(x)-f_{3}^{a^{\prime}, N}\left(x^{\prime}\right)\right| \leq \sup _{x \in K_{1}}\left|\hat{w}_{N}^{\hat{u}^{(N)}}(x)\right|\left\|\hat{P}^{a N}\left(x, K_{1} \cap \cdot\right)-\hat{P}^{a^{\prime} N}\left(x^{\prime}, K_{1} \cap \cdot\right)\right\|_{\text {var }} \\
+2 \varepsilon^{\frac{\varepsilon}{1+\varepsilon}}(M(K))^{\frac{1}{1+\varepsilon}} .
\end{array}
$$

For $\delta>0$ choose $K_{1}$ in (46) such that $\varepsilon^{\frac{\varepsilon}{1+\varepsilon}}(M(K))^{\frac{1}{1+\varepsilon}}<\frac{\delta}{4}$. For $x, x^{\prime} \in E_{0} \cup C_{1}$ and $a$, $a^{\prime} \in U$ such that

$$
\left\|\hat{P}^{a N}\left(x, C_{1} \cap \cdot\right)-\hat{P}^{a^{\prime} N}\left(x^{\prime}, C_{1} \cap \cdot\right)\right\|_{v a r} \leq \frac{\delta}{2\|c\|}
$$

and

$$
\left\|\hat{P}^{a N}\left(x, K_{1} \cap \cdot\right)-\hat{P}^{a^{\prime} N}\left(x^{\prime}, K_{1} \cap \cdot\right)\right\|_{v a r} \leq \frac{\delta}{2 \sup _{z \in K_{1}}\left|\hat{w}_{N}^{\hat{u}^{(N)}}(z)\right|}
$$

by (48)-(50) we obtain that

$$
\max _{i=1,2,3}\left|f_{i}^{a, N}(x)-f_{i}^{a^{\prime}, N}\left(x^{\prime}\right)\right| \leq \delta .
$$


Now by (A5) $\sup _{z \in K_{1}}\left|\hat{w}_{N}^{\hat{u}^{(N)}}(z)\right|$ is bounded in $N$ and therefore by (42) we can choose $x$, $x^{\prime}$ and $a, a^{\prime}$ in (51) and (52) uniformly in $N$, which completes the proof of equicontinuity.

Step 3. By step 1, 2 and (21) we immediately see that $E_{x}^{a, N}\left\{w_{N}^{\hat{u}^{(N)}}\left(x_{1}\right)\right\}$ is uniformly (in $N$ ) bounded and equicontinuous in $x$ and $a$ from compact subsets of $E \times U$. Since $\hat{u}^{(N)}$ is optimal for $P_{N}^{a}(x, d y)$ we have that $w_{N}^{\hat{u}_{N}}=w^{(N)}$. Therefore by Ascoli's theorem (thm. 33 of [8]) there is a subsequence $N_{k}$ such that $E_{x}^{a, N_{k}}\left\{w^{\left(N_{k}\right)}\left(x_{1}\right)\right\}$ converges uniformly in $a \in U$ and $x$ from compact subsets of $E$ and $\lambda^{\left(N_{k}\right)}\left(\hat{u}^{\left(N_{k}\right)}\right) \rightarrow \lambda\left(\right.$ since $\lambda^{N}\left(\hat{u}^{(N)}\right) \in$ $\left.\left[\inf _{x \in E, a \in U} c(x, a), \sup _{x \in E, a \in U} c(x, a)\right]\right)$. Consequently there is a continuous function $w$ such that

$$
w(x)=\inf _{a \in U}\left[c(x, a)-\lambda+\lim _{k \rightarrow \infty} \int_{E} w^{\left(N_{k}\right)}(y) P_{N_{k}}^{a}(x, d y)\right] .
$$

Moreover, since $w^{(N)}=w_{N}^{\hat{u}^{(N)}}$ is a solution to the suitable Bellman equation (9) (see Remark 1) we have that

$$
\begin{aligned}
w(x) & =\lim _{k \rightarrow \infty} \inf _{a \in U}\left[c(x, a)-\lambda+\int_{E} w^{\left(N_{k}\right)}(y) P_{N_{k}}^{a}(x, d y)\right] \\
& =\lim _{k \rightarrow \infty}\left(\lambda-\lambda^{\left(N_{k}\right)}\left(\hat{u}^{\left(N_{k}\right)}\right)+w^{\left(N_{k}\right)}(x)\right)=\lim _{k \rightarrow \infty} w^{\left(N_{k}\right)}(x) .
\end{aligned}
$$

Step 4. To prove that the function $w$ defined in (53) is a solution to the Bellman equation (45) it remains to show that

$$
\lim _{k \rightarrow \infty} E_{x}^{a, N_{k}}\left\{w^{\left(N_{k}\right)}\left(x_{1}\right)\right\}=E_{x}^{a}\left\{w\left(x_{1}\right)\right\} .
$$

In fact, by (A5) and Fatou's lemma

$$
E_{x}^{a}\left\{w\left(x_{1}\right)\right\} \leq \lim _{k \rightarrow \infty} E_{x}^{a, N_{k}}\left\{w^{\left(N_{k}\right)}\left(x_{1}\right)\right\}<\infty .
$$

By step 1 and 2 one can find a compact set $K_{1} \supset C$ such that

$$
\sup _{N} \sup _{a \in U} E_{x}^{a, N}\left\{1_{K_{1}^{c}}\left(x_{1}\right)\left|w^{(N)}\left(x_{1}\right)\right|\right\} \leq \frac{\varepsilon}{3}
$$

and

$$
\left.\sup _{a \in U} E_{x}^{a}\left\{1_{K_{1}^{c}}\left(x_{1}\right) \mid w\left(x_{1}\right)\right) \mid\right\} \leq \frac{\varepsilon}{3} .
$$

Therefore

$$
\begin{aligned}
&\left|E_{x}^{a}\left\{w\left(x_{1}\right)\right\}-E_{x}^{a, N_{k}}\left\{w^{\left(N_{k}\right)}\left(x_{1}\right)\right\}\right| \\
& \leq \mid\left|E_{x}^{a}\left\{1_{K_{1}}\left(x_{1}\right) w\left(x_{1}\right)\right\}-E_{x}^{a, N_{k}}\left\{1_{K_{1}}\left(x_{1}\right) w\left(x_{1}\right)\right\}\right| \\
&+\left|E_{x}^{a, N_{k}}\left\{1_{K_{1}}\left(x_{1}\right)\left(w\left(x_{1}\right)-w^{\left(N_{k}\right)}\left(x_{1}\right)\right)\right\}\right| \\
& \quad+E_{x}^{a, N_{k}}\left\{1_{K_{1}^{c}}\left(x_{1}\right) w^{\left(N_{k}\right)}\left(x_{1}\right)\right\}+E_{x}^{a}\left\{1_{K_{1}^{c}}\left(x_{1}\right) w\left(x_{1}\right)\right\} \\
& \leq \sup _{x \in K_{1}}|w(x)||| P^{a}\left(x, K_{1} \cap \cdot\right)-P^{a N}\left(x, K_{1} \cap \cdot\right) \|_{\text {var }}+\sup _{x \in K_{1}}\left|w(x)-w^{\left(N_{k}\right)}(x)\right|+\frac{2 \varepsilon}{3} .
\end{aligned}
$$

Consequently letting $k \rightarrow \infty$ and taking into account that $\varepsilon$ may be arbitrarily small we obtain the convergence (55). By continuity in $x$ and $a$ of the right hand side of (45) we have the existence of a Borel measurable function $\hat{u}$ for which the infimum is attained. 
Step 5. We shall show now that for Borel measurable $u: E \rightarrow U$ we have $\lambda(u) \geq \lambda$. In fact, then

$$
w(x) \leq c(x, u(x))-\lambda+\int_{E} w(y) P^{u(x)}(x, d y) .
$$

Define following (23)

$$
\hat{w}^{u}\left(x^{1}, x^{2}\right)=c\left(x^{1}, u\left(x^{1}\right)\right)-\lambda+\hat{E}_{x^{1}, x^{2}}^{u\left(x^{1}\right)}\left\{w\left(x_{1}^{1}\right)\right\} .
$$

Since by Corollary 1 for $a \in U$

$$
\begin{aligned}
E_{x}^{a}\left\{w\left(x_{1}\right)\right\} & =\hat{E}_{\delta_{x}^{*}}^{a}\left\{w\left(x_{1}^{1}\right)\right\} \\
& =1_{C}(x)\left[(1-\beta) \hat{E}_{(x, 0)}^{a}\left\{w\left(x_{1}^{1}\right)\right\}+\beta \hat{E}_{(x, 1)}^{a}\left\{w\left(x_{1}^{1}\right)\right\}\right]+1_{E \backslash C}(x) \hat{E}_{(x, 0)}^{a}\left\{w\left(x_{1}^{1}\right)\right\}
\end{aligned}
$$

from (59) we have

$$
\begin{aligned}
w(x) & \leq c(x, u(x))-\lambda+1_{C}(x)\left[(1-\beta) \hat{E}_{(x, 0)}^{u(x)}\left\{w\left(x_{1}^{1}\right)\right\}+\beta \hat{E}_{(x, 1)}^{u(x)}\left\{w\left(x_{1}^{1}\right)\right\}\right] \\
& +1_{E \backslash C}(x) \hat{E}_{(x, 0)}^{u(x)}\left\{w\left(x_{1}^{1}\right)\right\} \\
& =1_{C}(x)\left((1-\beta) \hat{w}^{u}(x, 0)+\beta \hat{w}^{u}(x, 1)\right)+1_{E \backslash C}(x) \hat{w}^{u}(x, 0) .
\end{aligned}
$$

Therefore

$$
\begin{aligned}
\hat{E}_{\left(x^{1}, x^{2}\right)}^{u\left(x^{1}\right)}\left\{w\left(x_{1}^{1}\right)\right\} & \leq \hat{E}_{\left(x^{1}, x^{2}\right)}^{u\left(x^{1}\right)}\left\{1_{C}\left(x_{1}^{1}\right)\left((1-\beta) \hat{w}^{u}\left(x_{1}^{1}, 0\right)+\beta \hat{w}^{u}\left(x_{1}^{1}, 1\right)\right)+1_{E \backslash C}\left(x_{1}^{1}\right) \hat{w}^{u}\left(x_{1}^{1}, 0\right)\right\} \\
& =\hat{E}_{\left(x^{1}, x^{2}\right)}^{\left(u\left(x^{1}\right)\right.}\left\{\hat{w}^{u}\left(x_{1}\right)\right\} .
\end{aligned}
$$

Consequently by (60) we have that

$$
\hat{w}^{u}\left(x^{1}, x^{2}\right) \leq c\left(x^{1}, u\left(x^{1}\right)\right)-\lambda+\hat{E}_{\left(x^{1}, x^{2}\right)}^{u\left(x^{1}\right)}\left\{\hat{w}^{u}\left(x_{1}\right)\right\} .
$$

Integrating both sides of the last inequality with respect to measure $\Psi^{u}$ we obtain that $\lambda \leq \lambda(u)$.

Step 6. By Proposition 1 and step 5 we have for any Borel measurable $u: E \rightarrow U$

$$
\lambda=\lambda(\hat{u})=J_{x}\left(\hat{u}\left(x_{n}\right)\right) \leq J_{x}\left(\left(u\left(x_{n}\right)\right)\right),
$$

which shows optimality of $\left(\hat{u}\left(x_{n}\right)\right)$ within the class of stationary controls. If for an admissible control $\left(a_{n}\right)$ we have $\limsup _{t \rightarrow \infty} \frac{1}{t} E_{x}^{\left(a_{n}\right)}\left\{w\left(x_{t}\right)\right\}=0$, then iterating (45) we obtain

$$
w(x) \leq E_{x}^{\left(a_{n}\right)}\left\{\sum_{i=0}^{t-1}\left(c\left(x_{i}, a_{i}\right)-\lambda\right)+w\left(x_{t}\right)\right\}
$$

and dividing both sides of the last inequality by $t$ and letting $t$ to infinity we obtain that $J_{x}\left(\left(a_{n}\right)\right) \geq \lambda$ which completes the proof.

7. Solution to multiplicative Bellman equation. Assume now that (A6) $\exists_{\epsilon>0}$ such that $\forall_{K \text { compact } \subset \hat{E}}$

$$
\sup _{a \in U} \sup _{x \in \hat{K}} \sup _{N} \hat{E}_{x}^{a, N}\left\{\exp \left\{\sum_{i=1}^{\tau_{C_{1}}} \gamma\left(c\left(x_{i}^{1}, \hat{u}_{\gamma}^{(N)}\left(x_{i}^{1}\right)\right)-\lambda_{\gamma}^{(N)}\left(\hat{u}_{\gamma}^{(N)}\right)\right)\right\}\right\}^{1+\epsilon}<\infty,
$$

where the control at the first time is $a_{0}=a$ and $a_{n}=u_{N}\left(x_{n}^{1}\right)$ for $n \geq 1$. 
A sufficient condition for (A6) can be found in [3]. We can now recall Theorem 1 of [3]: THEOREM 2. Under (A1)-(A4) and (A6) there exist $\lambda_{\gamma}$ and a continuous function $w_{\gamma}$ : $E \rightarrow \mathbb{R}$ such that

$$
e^{w_{\gamma}(x)}=\inf _{a \in U}\left[e^{\gamma c(x, a)-\lambda_{\gamma}} \int_{E} e^{w_{\gamma}(y)} P^{a}(x, d y)\right]
$$

Moreover, under:

(D2) $\sup _{N} \sup _{\left(a_{n}\right) \in \mathcal{U}_{s}} \hat{E}_{x}^{\left(a_{n}\right), N}\left\{\exp \left\{\gamma\|c\|_{s p} \tau_{C_{1}}\right\}\right\}<\infty$ for $x \in C_{1}$

(D3) $\sup _{\left(a_{n}\right) \in \mathcal{U}_{s}} \hat{E}_{x}^{\left(a_{n}\right)}\left\{\exp \left\{(1+\varepsilon) \gamma\|c\|_{s p} \tau_{C_{1}}\right\}\right\}<\infty$ for $x \in C_{1}$ and a sufficiently small $\varepsilon>0$,

we have that

$$
\lambda_{\gamma}=\inf _{\left(a_{n}\right) \in \mathcal{U}_{s}} J_{\gamma}\left(\left(a_{n}\right)\right)=\lim _{N \rightarrow \infty} \lambda_{\gamma}^{(N)}\left(\hat{u}_{\gamma}^{(N)}\right) .
$$

Assuming additionally that (D1) is satisfied for $\hat{a}_{n}=\hat{u}\left(x_{n}\right)$, where $\hat{u}$ is a Borel measurable function for which the infimum in the right hand side of (50) is attained we have that $\lambda_{\gamma}=\lambda_{\gamma}(\hat{u})$. Furthermore, if for an admissible control $\left(a_{n}\right)$ we have

$$
\limsup _{t \rightarrow \infty} E_{x}^{\left(a_{n}\right)}\left\{\left(E_{x_{t}}^{a_{t}}\left\{e^{w\left(x_{1}\right)}\right\}\right)^{\alpha}\right\}<\infty
$$

for every $\alpha>1$, then $\lambda_{\gamma} \leq J_{\gamma}\left(\left(a_{n}\right)\right)$.

8. Asymptotics of Bellman equations. Notice first that by Proposition 3 if (D1) is satisfied for sufficiently small $\gamma>0$, we have for any Borel measurable $u: E \rightarrow U$

$$
J_{\gamma}((u(x(n))) \rightarrow J((u(x(n))))
$$

as $\gamma \rightarrow 0$, and the limit is decreasing. Consequently we have

THEOREM 3. Under (A1)-(A6) if (D1)-(D3) are satisfied for sufficiently small $\gamma>0$ we have

$$
\lim _{\gamma \rightarrow 0} \lambda_{\gamma}=\lambda .
$$

Furthermore, risk neutral $\varepsilon$-optimal control $u \in \mathcal{U}_{s}$ within the class of stationary controls is nearly optimal for the risk sensitive cost functional with $\gamma$ close to 0 , within the class of stationary controls.

Proof. By Theorem 2

$$
\lambda_{\gamma}=\inf _{u \in \mathcal{U}_{s}} J_{\gamma}(u)
$$

and by Theorem 1

$$
\lambda=\inf _{u \in \mathcal{U}_{s}} J(u) .
$$

Therefore from (66) we immediately obtain (67). Now, if $u \in \mathcal{U}_{s}$ is $\varepsilon$-optimal for $J$ within the class of stationary strategies, then by (66) for $0<\gamma<\gamma_{0}$ we have

$$
J_{\gamma}(u) \leq J(u)+\varepsilon \leq \lambda+2 \varepsilon \leq \lambda_{\gamma}+3 \varepsilon,
$$

which is the required $3 \varepsilon$-optimality. 
REMARK 2. Although we have convergence (67) it is not clear that the function $w_{\gamma}$, the solution to (64) converges, (or at least a suitable subsequence does) to the function $w$, the solution to (45), as $\gamma \rightarrow 0$, provided that at fixed point $\bar{x} \in E$ we have $w_{\gamma}(\bar{x})=w(\bar{x})$.

\section{References}

[1] T. R. Bielecki and S. Pliska, Risk sensitive dynamic asset management, JAMO 39 (1999), 337-360.

[2] G. B. Di Masi and Ł. Stettner, Risk sensitive control of discrete time Markov processes with infinite horizon, SIAM J. Control Optimiz. 38 (2000), 61-78.

[3] G. B. Di Masi and Ł. Stettner, Infinite horizon risk sensitive control of discrete time Markov processes under minorization property, SIAM J. Control Optimiz., submitted.

[4] G. B. Di Masi and E. Stettner, Remarks on risk neutral and risk sensitive portfolio optimization, in: The Shiryaev Festschrift, From Stochastic Calculus to Mathematical Finance, Yu. Kabanov, R. Liptser, J. Stoyanov (eds.), Springer, 2006, 211-226.

[5] I. Kontoyiannis and S. P. Meyn, Spectral theory and limit theorems for geometrically ergodic Markov processes, Ann. Appl. Prob. 13 (2003), 304-362.

[6] S. P. Meyn and R. L. Tweedie, Markov Chains and Stochastic Stability, Springer, 1996.

[7] H. Pham, A large deviations approach to optimal long term investment, Finance Stoch. 7 (2003), 169-195.

[8] H. L. Royden, Real Analysis, Mac Millan, New York, 1968.

[9] E. Stettner, On the Poisson equation and optimal stopping of ergodic Markov processes, Stochastics 18 (1986), 25-48. 